\section{THE ANTI-TUMOR ACTIVITY OF HSP-90 THERAPEUTIC CANCER VACCINE (AST-021P) COMBINE WITH TLR2/3 AGONIST IN A MMTV-NEU TRANSGENIC MODEL}

${ }^{1}$ Jinho Kang, ${ }^{2}$ Eunkyo Joung, ${ }^{3}$ Hunwoo Shin, ${ }^{4}$ Byung cheol Ahn, ${ }^{4}$ Eunjung Jung, ${ }^{3}$ Hun Jung*, ${ }^{1}$ Kyong Hwa Park. ${ }^{1}$ Korea University College of Medicine, Seoul, Korea, Republic of; ${ }^{2}$ Aston Sci. Inc, Seoul, Korea, Republic of; ${ }^{3}$ Aston Sci. Inc., Seoul, Korea, Republic of; ${ }^{4} \mathrm{CHA}$ Vaccine Institute, Seoul, Korea, Republic of

Background AST-021p, which is derived from HLA class II binding epitopes of human HSP90 protein, is an investigational therapeutic cancer vaccine for the malignant neoplasms. AST-021p is designed to demonstrate the immunologic efficacy by activating antigen-specific CD4+ Th1 cell in humans. Due to their ability to link the innate with the adaptive immune response, Toll-like receptor (TLR) agonists are highly promising as adjuvants in vaccines against life-threatening and complex diseases such as cancer, AIDS and malaria. In this study, AST-021p was investigated to evaluate the immunogenicity and tumor growth inhibitory effect under the condition of combining with various immune adjuvants derived from TLR agonists, using in-vivo model.

Methods Three different agonists of TLR (TLR-4, TLR-2/3, TLR-7/8) were assigned to investigate the immunogenicity in each group (4 FVB mice/group, total 4 groups). AST-021p was intradermally injected 3 times with different TLR-agonists and the immunogenicity was assessed from mouse splenocyte by HSP90-specific IFN- $\gamma$ ELISpot method. We also examined the efficacy of AST-021p and selected TLR-agonist in MMTVneu Tg mice (4 mice/group, conducted twice and A total 8 mice was assigned to each group). The combination of AST-021p and TLR-2/3 agonist (AST-021p plus TLR-2/3 agonist) was injected 3 times every 10 days to mice followed by inoculated mouse mammary cancer cell line. The tumor volume change and immunogenicity were evaluated.

Results The most effective TLR-agonist as a potent immune adjuvant was a TLR-2/3 agonist (L-pampoTM, supplied by CHA Vaccine Institute). In MMTV-Neu transgenic mice, AST021p $(100 \mu \mathrm{g})$ plus TLR-2/3 agonist significantly enhanced immunogenicity by increasing up to $130 \pm 10$ HSP-90 epitope specific $\mathrm{T}$ cells per $1 \times 105$ splenocytes $(\mathrm{P}<0.001)$. AST-021p plus TLR-2/3 agonist also showed higher tumor growth inhibitory effect $(170 \pm 108 \mathrm{~mm} 3)$ on post-implantation 35 th day by suppressing mouse mammary cancer cell line $(5 \times 105)$-derived tumor growth, compared with a TLR-2/3 agonist alone (1031 $\pm 450 \mathrm{~mm} 3$ ).

Conclusions Combination regimen of AST-021p and TLR-2/3 agonist (as immune adjuvant) demonstrated significant immunogenicity and tumor prevention effect in in-vivo study. These data supported the clinical study of AST-021p combined with TLR-2/3 agonist as active immune adjuvant in certain tumor types, and phase $1 / 2$ clinical program would be expected to be initiated.

Acknowledgements Not applicable

Trial Registration Not applicable

\section{REFERENCES}

1. Csermely P, Schnaider T, Soti C, Prohaszka Z, Nardai G. The 90-kDa molecular chaperone family: structure, function, and clinical applications. A comprehensive review. Pharmacol Ther 1998;79,129-168.

2. Wang $H$, Lu M, Yao M, Zhu W. Effects of treatment with an Hsp90 inhibitor in tumors based on 15 phase II clinical trials. Mol Clin Oncol 2016:5,326-334.

3. Ramalingam S, Goss G, Rosell R. Schmid-Bindert G, Zaric B, Andric Z, Bondarenko I, Komov D, Ceric T, Khuri F. A randomized phase II study of ganetespib, a heat shock protein 90 inhibitor, in combination with docetaxel in second-line therapy of advanced non-small cell lung cancer (GALAXY-1). Ann Oncol Off J Eur Soc Med Oncol 2015,26,1741-1748.
Ethics Approval All experimental procedures involving mice were performed with the guidance protocols approved by the Institutional Animal Care and Use Committee of Korea University (IACUC, Approval number: KOREA-2019-129)

Consent It is not an abstract containing sensitive or identifiable information.

http://dx.doi.org/10.1136/jitc-2021-SITC2021.860 